\title{
Aborto e estigma: uma análise da produção científica sobre a temática
}

\author{
Abortion and stigma: \\ an analysis of the scientific literature on the theme
}

Leila Adesse ${ }^{1}$

Claudia Bonan Jannotti ${ }^{1}$

Katia Silveira da Silva ${ }^{1}$

Vania Matos Fonseca ${ }^{1}$
${ }^{1}$ Pós-Graduação em Saúde da Mulher e da Criança, Instituto de Saúde da Mulher, da Criança e do Adolescente Fernandes Figueira, Fundação Oswaldo Cruz. Av. Rui Barbosa 716, Flamengo. 22250-020 Rio de Janeiro RJ Brasil.

leila.adesse@gmail.com
Abstract This article analyzes the scientific output on abortion and social stigma and the potential of the stigma category for abortion care in Brazil. An integrative review of publications on scientific databases without a time limit was conducted. Sixty-five publications with the social representations of women who had an abortion and the professionals who attended them were analyzed. The obstacles to the implementation of abortion laws and the protocols and norms that facilitate access to quality services care was explored. The conceptual relevance of Erving Goffman was a key element for the understanding of the gender transgression stereotypes, the imperative of motherhood, sexual purity, which tarnishes women who have abortions as inferior, damaged, promiscuous, sinful and murderous. Groups most affected by stigmatization were identified, namely women having an abortion and health professionals. Female conflicts due to the duality of concealing/revealing their abortions, the professional's conscientious objection and the obstacles facing the implementation of public policies emerged from the study. Reflecting on the role of stigma may interfere in the cycle of clandestine abortion and contribute to the (re)design of interventions that help to reduce damage to the sexual and reproductive health of women.

Key words Abortion, Social Stigma, Health care
Resumo O artigo objetiva analisar a produção científica sobre aborto e estigma social e o potencial da categoria estigma para estudos sobre a assistência ao abortamento no Brasil. Utilizou-se o método de revisão integrativa de publicações das bases científicas, optando por não estabelecer limite temporal. Analisou-se 65 publicações com as representações sociais de mulheres que abortame de profissionais que as atendem; exploram os obstáculos para a implementação das leis do aborto e dos protocolos e normas que facilitariam o acesso a serviços de qualidade. À relevância conceitual de Erving Goffman somou-se a compreensão sobre a transgressão dos estereótipos de gênero, o imperativo da maternidade, a pureza sexual, que marca as mulheres que abortam como seres inferiores, deteriorados: promíscuas, pecadoras, assassinas. Identificaram-se grupos mais afetados pela estigmatização: mulheres em abortamento, profissionais de saúde. O conflito feminino pela dualidade ocultar/revelar seus abortamentos, a objeção de consciência de profissionais e os obstáculos na implementação de políticas públicas emergiram dos trabalhos. A reflexão sobre o papel do estigma pode interferir no ciclo do aborto clandestino e contribuir para o (re) desenho de intervenções que apoiem a redução de danos à saúde sexual e reprodutiva das mulheres.

Palavras-chave Aborto, Estigma social, Assistência à saúde 


\section{Introdução}

Há por parte da comunidade internacional um claro entendimento da necessidade de incluir, nas políticas públicas, medidas para redução da mortalidade materna por aborto inseguro. Os esforços de diferentes organismos internacionais para ampliar o acesso a serviços de atenção pós-abortamento e apoiar a revisão das leis que incriminam as mulheres que abortam e os profissionais de saúde que prestam atendimento têm esbarrado em múltiplos obstáculos - um desses é o estigma ${ }^{1}$.

As análises conceituais sobre o estigma em situações ligadas à saúde têm atraído atenção de pesquisadores e profissionais pelo fato deste contribuir como um fardo oculto e influenciar a efetividade do cuidado, tornando-se um problema de saúde pública. O trabalho pioneiro de Erving Goffman ${ }^{2}$ abordou o estigma associado a doenças incapacitantes, distúrbios mentais e doenças infecto-contagiosas. A concepção do estigma como um atributo meramente físico tem, progressivamente, sido revista e ampliada, movendo-se do nível individual para uma raiz mais social. Transcendendo a ideia de uma "marca" física e visível, duas perspectivas analíticas se destacam: nos estudos sociológicos e antropológicos refere-se ao processo inerente à interação social estabelecida pelo estigmatizado e por quem estigmatiza através de categorias como rotulação, status social, desviante e normal; nas perspectivas mais psicológicas atribuindo-se uma maior ênfase a processos psicossociais e reações sociais preconceituosas $^{3}$. Em função dessa ampliação teórica do conceito, em 2011, quando o Medical Subjects Headings (MeSH) cria um descritor com a palavra "estigma”, este é definido como "social stigma". No presente trabalho, os termos estigma e estigma social serão utilizados de modo indiferenciado, mas sempre ancorados em perspectivas teórico-analíticas interdisciplinares que vêm do campo das ciências sociais e humanas.

Pesquisas no âmbito do HIV/AIDS têm demonstrado a utilidade da categoria estigma social para análises críticas da prática clínica, promovendo novos conhecimentos sobre o cuidado e mudanças de atitudes e compromissos éticos dos profissionais e gestores de saúde ${ }^{4}$. Artigos que analisam a assistência às mulheres em abortamento nos serviços públicos brasileiros têm demonstrado que elas primam pela baixa qualidade, maus tratos e discriminações ${ }^{5}$. Adicionalmente, muitas das denúncias contra mulheres que praticaram aborto inseguro são feitas por profissionais de saúde, tornando-se uma barreira ao acesso aos cuidados.
O objetivo deste artigo é analisar a produção científica sobre aborto e estigma social e discutir o potencial da categoria estigma para os estudos sobre os processos de assistência às mulheres em situação de abortamento, em nosso país.

\section{Materiais e métodos}

Utilizou-se o método de revisão integrativa ${ }^{6}$ com o levantamento de trabalhos nas bases de dados Science Direct Scopus (Scopus), Literatura Latino Americana e do Caribe em Ciências da Saúde (Lilacs) e Medical Literature Analysis and Retrieval Sistem (Medline) - essas duas últimas, por meio da Biblioteca Virtual de Saúde (BVS). Optou-se por analisar somente trabalhos publicados em periódicos indexados como artigos, notas, revisões e editoriais. Foram incluídos trabalhos em língua portuguesa, inglesa e espanhola. Nenhum estudo em francês foi encontrado. Optou-se por não estabelecer limite temporal à pesquisa e o levantamento dos trabalhos estendeu-se até 30 de agosto de 2014.

O critério de busca foi a combinação dos termos "aborto" e "estigma social" (usado como descritor e palavra chave), em português e inglês, com o operador boleano and. Foram excluídos trabalhos cujas áreas ou assuntos principais não eram relacionados ao tema estudado.

Selecionamos 165 publicações (113 no Scopus e 52 no Medline). Após a eliminação das duplicatas, chegou-se a 108. Com a leitura dos resumos, foram excluídos 39 trabalhos não correlatos à temática, constituindo uma seleção de 69 artigos. Após a leitura na íntegra, quatro foram excluídos por tratarem o tema do aborto marginalmente, e 65 publicações compuseram o corpus do estudo. A Figura 1 apresenta esquematicamente as fases da revisão bibliográfica.

\section{Resultados e discussão}

A Tabela 1 apresenta a distribuição dos trabalhos segundo o ano de publicação, a região geográfica onde foram realizados, os perfis profissionais dos primeiros autores e a nacionalidade das instituições de origem.

O levantamento das publicações sem restrição temporal permitiu a observação da evolução da série histórica. O primeiro artigo encontrado é de 1972, o segundo é de 1984, e a temática reaparece somente em 1999, em dois artigos. De 2002 a 2010, foram selecionados vinte e quatro artigos e entre 2011 e 2014, houve uma proliferação dos estudos sobre aborto e estigma, com 
Tabela 1. Distribuição dos trabalhos por ano de publicação, contexto geográfico, perfil profissional do primeiro autor e a nacionalidade da instituição de filiação $(n=65)$.

\begin{tabular}{lc}
\hline & Quantidade \\
\hline Ano de publicação & \\
1972 & 1 \\
1984 & 1 \\
1999 & 2 \\
$2002-2010$ & 24 \\
$2011-2014$ & 37 \\
Contexto geográfico & \\
América do Norte & 20 \\
África & 13 \\
América Latina e Caribe & 10 \\
Ásia & 8 \\
Europa & 3 \\
Oceania & 1 \\
Multicontinental (dados primários) & 3 \\
Multicontinental (dados secundários) & 7 \\
Perfil profissional dos autores & \\
Profissionais de saúde & 19 \\
Saúde Pública & 19 \\
Ciências Sociais e Humanas & 27 \\
Nacionalidade das instituições & \\
América do Norte & 38 \\
África & 5 \\
América Latina e Caribe & 8 \\
Ásia & 3 \\
Europa & 9 \\
Oceania & \\
\hline
\end{tabular}

trinta e sete publicações. O aumento deste último período coincide com a entrada do termo "estig- ma social" como um descritor nas bases científicas (Tabela 1).

A quase totalidade das publicações foi escrita em inglês, entretanto os estudos se referem a realidades de diferentes regiões do mundo: em contextos da América do Norte lideraram com vinte trabalhos; e dos dez latino-americanos apenas dois no Brasil.

Quanto ao perfil profissional dos primeiros autores, eles se distribuem homogeneamente em três grandes áreas do conhecimento: profissionais de saúde, pesquisadores em saúde pública e cientistas sociais. Mais da metade dos autores é filiada a instituições de pesquisa norte-americanas (38): destes, a organização Ipas responde por 11 trabalhos, a pesquisadora Lisa Harris da Universidade de Michigan é primeira autora de cinco artigos e pesquisadoras da Universidade da Califórnia assinam seis. Na Europa, instituições do Reino Unido lideraram, com seis publicações, e na América Latina, grupos mexicanos, com igual quantidade.

No conjunto de trabalhos analisados, a relação entre aborto e estigma é abordada desde uma perspectiva crítica, apontando que, no campo social, médico e jurídico, mesmo em contextos com leis e protocolos mais liberais, a questão do abortamento encontra-se ainda circundada por preconceitos e discriminações.

No Quadro 1 são apresentadas as publicações referentes ao período 2011-2014 com informações sobre autores, o ano de publicação, o título, o desenho metodológico e o objeto/objetivos.

Para análise do conteúdo temático os trabalhos foram reunidos em três grupos: o primeiro reúne aquelas que se concentram na discussão teórico-conceitual sobre a categoria estigma so- 
Quadro 1. Características dos artigos sobre estigma e aborto no período de 2011-2014.

\begin{tabular}{|c|c|c|c|}
\hline Autor e Ano & Título & $\begin{array}{l}\text { Tipo de pesquisa/ } \\
\text { Método }\end{array}$ & Objeto/Objetivo de Estudo \\
\hline $\begin{array}{l}\text { Cook R, Dickens BM, } \\
2014^{7}\end{array}$ & $\begin{array}{l}\text { Reducing stigma in } \\
\text { reproductive health }\end{array}$ & $\begin{array}{l}\text { Qualitativa /Análise } \\
\text { conceitual }\end{array}$ & $\begin{array}{l}\text { Examinar o papel das leis e das } \\
\text { Instituições no estigma do aborto }\end{array}$ \\
\hline Lamas M, 2014 & $\begin{array}{l}\text { Between the stigma and the } \\
\text { law }\end{array}$ & Qualitativa & $\begin{array}{l}\text { Identificar as lacunas na implementação } \\
\text { de leis e políticas de saúde reprodutiva }\end{array}$ \\
\hline Faundes et al., $2013^{9}$ & $\begin{array}{l}\text { Conscientious objection or } \\
\text { fear of social stigma? }\end{array}$ & $\begin{array}{l}\text { Qualitativa/ Análise } \\
\text { conceitual }\end{array}$ & $\begin{array}{l}\text { Examina a objeção de consciência } \\
\text { como reação do profissional de temer a } \\
\text { estigmatização }\end{array}$ \\
\hline Kumar A, $2013^{10}$ & Everything is not stigma & Análise conceitual & $\begin{array}{l}\text { Analisar as pesquisas qualitativas e a } \\
\text { "explosão" conceitual do estigma }\end{array}$ \\
\hline Cockrill K, Nock A, $2013^{11}$ & $\begin{array}{l}\text { I'm not that type of person. } \\
\text { Managing the Stigma of } \\
\text { having an abortion }\end{array}$ & $\begin{array}{l}\text { Pesquisa com dados } \\
\text { secundários }\end{array}$ & $\begin{array}{l}\text { Estudar os efeitos da estigmatização para } \\
\text { as mulheres que abortam }\end{array}$ \\
\hline Cockrill K et al., 2013 ${ }^{12}$ & $\begin{array}{l}\text { Stigma of abortion: } \\
\text { Development of a scale and } \\
\text { characteristics of women } \\
\text { experiencing abortion } \\
\text { stigma }\end{array}$ & Qualitativa & $\begin{array}{l}\text { Desenvolver e aplicar uma escala para } \\
\text { tipificar o estigma individual }\end{array}$ \\
\hline Payne CM et al., $2013^{13}$ & $\begin{array}{l}\text { Why women are dying from } \\
\text { unsafe abortion: narratives } \\
\text { of Ghanaian abortion } \\
\text { providers }\end{array}$ & Qualitativa & $\begin{array}{l}\text { Estudar os efeitos do estigma para } \\
\text { profissionais de saúde }\end{array}$ \\
\hline Norman WV et al., $2013^{14}$ & $\begin{array}{l}\text { Barriers to rural induced } \\
\text { abortion services in } \\
\text { Canada: findings of the } \\
\text { British Columbia Abortion } \\
\text { Providers }\end{array}$ & Qualitativa & $\begin{array}{l}\text { Identificar as barreiras institucionais e } \\
\text { dos profissionais na assistência }\end{array}$ \\
\hline Dressler J, $2013^{15}$ & $\begin{array}{l}\text { The Perspective of rural } \\
\text { physicians providing } \\
\text { abortion in Canada: } \\
\text { qualitative findings of the } \\
\text { BC } \\
\text { Abortion Providers Survey } \\
\text { (BCAPS) }\end{array}$ & Qualitativa & $\begin{array}{l}\text { Estudar os efeitos da estigmatização para } \\
\text { profissionais de saúde }\end{array}$ \\
\hline Khan A, $2013^{16}$ & $\begin{array}{l}\text { Induced abortion in } \\
\text { Pakistan: community based } \\
\text { research }\end{array}$ & $\begin{array}{l}\text { Revisão de pesquisas } \\
\text { qualitativas }\end{array}$ & $\begin{array}{l}\text { Prover um perfil das mulheres que } \\
\text { abortam, identificar suas razões e } \\
\text { condições do aborto }\end{array}$ \\
\hline Umuhoza C et al., $2013^{17}$ & $\begin{array}{l}\text { Advocating for safe abortion } \\
\text { in Rwanda: How young } \\
\text { peopleand the personal } \\
\text { stories of young women in } \\
\text { prison brought } \\
\text { about change }\end{array}$ & Qualitativa & $\begin{array}{l}\text { Analisar estratégias politicas (advocacy) } \\
\text { para revisão da lei do aborto }\end{array}$ \\
\hline
\end{tabular}


Quadro 1. continuação

\begin{tabular}{|c|c|c|c|}
\hline Autor e Ano & Título & $\begin{array}{c}\text { Tipo de pesquisa/ } \\
\text { Método }\end{array}$ & Objeto/Objetivo de Estudo \\
\hline $\begin{array}{l}\text { Culwell KR, Hurwitz M, } \\
2013^{18}\end{array}$ & $\begin{array}{l}\text { Addressing Barriers to safe } \\
\text { abortion }\end{array}$ & Análise conceitual & $\begin{array}{l}\text { Avaliar o impacto do aborto na } \\
\text { mortalidade materna; barreiras e estigma }\end{array}$ \\
\hline $\begin{array}{l}\text { Harris LH et al., } \\
2013^{19}\end{array}$ & $\begin{array}{l}\text { Physicians, abortion } \\
\text { provision and the Legitimacy } \\
\text { paradox }\end{array}$ & Reflexão conceitual & $\begin{array}{l}\text { Discutir os conflitos do profissional } \\
\text { na assistência ao aborto e o estigma de } \\
\text { colegas de profissão e pela sociedade }\end{array}$ \\
\hline Smith S, $2013^{20}$ & $\begin{array}{l}\text { Reproductive health and the } \\
\text { question of abortion in } \\
\text { Botswana: a review }\end{array}$ & Qualitativa & $\begin{array}{l}\text { Analisar o estigma segundo a abordagem } \\
\text { de gênero e fatores sócio-culturais }\end{array}$ \\
\hline $\begin{array}{l}\text { Astbury-Ward E et al., } \\
2012^{21}\end{array}$ & $\begin{array}{l}\text { Stigma, Abortion, and } \\
\text { Disclosure-Findings from a } \\
\text { Qualitative Study }\end{array}$ & Reflexão conceitual & $\begin{array}{l}\text { Analisar os grupos afetados pelo estigma } \\
\text { em consequência da revelação do aborto }\end{array}$ \\
\hline Harris LH, $2012^{22}$ & $\begin{array}{l}\text { Stigma and abortion } \\
\text { complications in the United } \\
\text { States }\end{array}$ & Reflexão conceitual & $\begin{array}{l}\text { Analisar em profundidade dois casos } \\
\text { de assistência prestada por profissionais } \\
\text { como estratégia de advocacy }\end{array}$ \\
\hline Tong W et al., $2012^{23}$ & $\begin{array}{l}\text { Exploring pregnancy } \\
\text { termination experiences } \\
\text { and needs among Malaysian } \\
\text { women: A qualitative study }\end{array}$ & Qualitativa & $\begin{array}{l}\text { Identificar necessidades das mulheres que } \\
\text { abortam para apoiar implementação de } \\
\text { políticas públicas }\end{array}$ \\
\hline Banerjee SK et al., $2012^{24}$ & $\begin{array}{l}\text { Woman-centered research } \\
\text { on access to safe abortion } \\
\text { services nd implications } \\
\text { for behavioral change } \\
\text { communication } \\
\text { interventions: A cross- } \\
\text { sectional study of women in } \\
\text { Bihar and Jharkhand, India }\end{array}$ & $\begin{array}{l}\text { Quantitativo } \\
\text { epidemiológico } \\
\text { seccional }\end{array}$ & $\begin{array}{l}\text { Analisar experiências de mulheres para } \\
\text { acessar assistência ao aborto e apoiar } \\
\text { campanhas de mídia para mudanças de } \\
\text { atitude da sociedade }\end{array}$ \\
\hline De Zordo S, $2012^{25}$ & $\begin{array}{l}\text { Representações e } \\
\text { experiências sobre } \\
\text { aborto legal e ilegal dos } \\
\text { ginecologista-obstetras } \\
\text { trabalhando em } 2 \\
\text { maternidades em Salvador, } \\
\text { Bahia }\end{array}$ & Qualitativa & $\begin{array}{l}\text { Analisar as narrativas de conflitos dos } \\
\text { profissionais na assistência ao aborto } \\
\text { legal e provocado }\end{array}$ \\
\hline $\begin{array}{l}\text { Shellenberg KM, Tsui AO, } \\
2012^{26}\end{array}$ & $\begin{array}{l}\text { Correlates of perceived and } \\
\text { internalized stigma among } \\
\text { abortion patients in the US } \\
\text { USA: an exploration by } \\
\text { race and Hispanic ethnicity }\end{array}$ & $\begin{array}{l}\text { Quantitativo } \\
\text { epidemiológico } \\
\text { seccional }\end{array}$ & $\begin{array}{l}\text { Estimar o estigma percebido e } \\
\text { internalizado pelas mulheres que } \\
\text { abortam e a relação com raça/etnia }\end{array}$ \\
\hline $\begin{array}{l}\text { McMurtie SM, García S, } \\
2012^{27}\end{array}$ & $\begin{array}{l}\text { Public opinion about } \\
\text { abortion-related stigma } \\
\text { among Mexican Catholics } \\
\text { and implications for unsafe } \\
\text { abortion }\end{array}$ & Qualitativo & $\begin{array}{l}\text { Analisar a representação social do aborto } \\
\text { entre mulheres e homens de religião } \\
\text { católica }\end{array}$ \\
\hline
\end{tabular}




\begin{tabular}{|c|c|c|c|}
\hline \multicolumn{4}{|l|}{ Quadro 1. continuação } \\
\hline Autor e Ano & Título & $\begin{array}{l}\text { Tipo de pesquisa/ } \\
\text { Método }\end{array}$ & Objeto/Objetivo de Estudo \\
\hline $\begin{array}{l}\text { Levandowiski BA et al., } \\
2012^{28}\end{array}$ & $\begin{array}{l}\text { Investigating social } \\
\text { consequences of unwanted } \\
\text { pregnancy and unsafe } \\
\text { abortion in Malawi: the role } \\
\text { of stigma }\end{array}$ & Qualitativo & $\begin{array}{l}\text { Analisar a percepção e mitos de mulheres } \\
\text { e homens sobre anticoncepção e aborto }\end{array}$ \\
\hline $\begin{array}{l}\text { Hosseini-Chavosshi M et } \\
\text { al., 2012 }\end{array}$ & $\begin{array}{l}\text { Social and psychological } \\
\text { consequences of abortion } \\
\text { in Iran. }\end{array}$ & Qualitativo & $\begin{array}{l}\text { Analisar a morbimortalidade do aborto } \\
\text { segundo aspectos sociais }\end{array}$ \\
\hline O’Donnell J et al., $2011^{30}$ & $\begin{array}{l}\text { Resistance and vulnerability } \\
\text { to stigmatization in } \\
\text { abortion work }\end{array}$ & Reflexão conceitual & Profissionais e superação do estigma \\
\hline Gipson JD et al., $2011^{31}$ & $\begin{array}{l}\text { Perceptions and practices } \\
\text { of illegal abortion among } \\
\text { urban young adults in the } \\
\text { Philippines: A qualitative } \\
\text { study }\end{array}$ & Qualitativa & $\begin{array}{l}\text { Identificar os relatos de jovens na busca } \\
\text { por aborto clandestino }\end{array}$ \\
\hline Harris LH et al., $2011^{32}$ & $\begin{array}{l}\text { Dynamics of stigma in } \\
\text { abortion work: Findings } \\
\text { from a pilot study of the } \\
\text { Providers Share Workshop }\end{array}$ & Qualitativo & $\begin{array}{l}\text { Identificar os relatos de estigma de } \\
\text { profissionais de saúde na assistência ao } \\
\text { aborto }\end{array}$ \\
\hline $\begin{array}{l}\text { Harris LH, Grossman D, } \\
2011^{33}\end{array}$ & $\begin{array}{l}\text { Confronting challenge of } \\
\text { unsafe second- trimester } \\
\text { abortion }\end{array}$ & Análise conceitual & $\begin{array}{l}\text { Analisar conflitos éticos dos profissionais } \\
\text { de saúde nos casos de aborto no } 2^{\circ} \\
\text { trimestre de gestação }\end{array}$ \\
\hline $\begin{array}{l}\text { Shellenberg KM et al., } \\
2011^{34}\end{array}$ & $\begin{array}{l}\text { Social stigma and disclosure } \\
\text { about induced abortion: } \\
\text { Results from an exploratory } \\
\text { study }\end{array}$ & $\begin{array}{l}\text { Qualitativa } \\
\text { Multicêntrico }\end{array}$ & $\begin{array}{l}\text { Comparar a percepção de mulheres sobre } \\
\text { estigma na revelação do abortamento }\end{array}$ \\
\hline Tsui AO et al., $2011^{35}$ & $\begin{array}{l}\text { Managing unplanned } \\
\text { pregnancies in five countries: } \\
\text { Perspectives on } \\
\text { contraception and abortion } \\
\text { decisions }\end{array}$ & $\begin{array}{l}\text { Qualitativa } \\
\text { Multicêntrico }\end{array}$ & $\begin{array}{l}\text { Analisar a percepção de mulheres sobre } \\
\text { contracepção e aborto }\end{array}$ \\
\hline Palomino $\mathrm{N}$ et al., $2011^{36}$ & $\begin{array}{l}\text { The social constructions of } \\
\text { unwanted pregnancy and } \\
\text { abortion in Lima, Peru }\end{array}$ & Qualitativa & $\begin{array}{l}\text { Analisar a percepção das mulheres sobre } \\
\text { gravidez indesejada e aborto }\end{array}$ \\
\hline Omidey AK, $2011^{37}$ & $\begin{array}{l}\text { Contraceptive practice, } \\
\text { unwanted pregnancies and } \\
\text { induced abortion in } \\
\text { Southwest, Nigeria }\end{array}$ & Qualitativa & $\begin{array}{l}\text { Analisar a percepção das mulheres sobre } \\
\text { gravidez indesejada e aborto }\end{array}$ \\
\hline Rodríguez JV et al., $2011^{38}$ & $\begin{array}{l}\text { Public Opinion on Abortion } \\
\text { in Eight Mexican States amid } \\
\text { Opposition to Legalization }\end{array}$ & Pesquisa de opinião & $\begin{array}{l}\text { Comparar a percepção da população } \\
\text { de oito estados sobre a reforma legal no } \\
\text { México, DF }\end{array}$ \\
\hline
\end{tabular}




\begin{tabular}{|c|c|c|c|}
\hline \multicolumn{4}{|l|}{ Quadro 1. continuação } \\
\hline Autor e Ano & Título & $\begin{array}{l}\text { Tipo de pesquisa/ } \\
\text { Método }\end{array}$ & Objeto/Objetivo de Estudo \\
\hline Bateman C, $2011^{39}$ & $\begin{array}{l}\text { Abortion practices } \\
\text { undermining reformist laws } \\
\text { - experts }\end{array}$ & Nota analítica & $\begin{array}{l}\text { Apresentar as barreiras para } \\
\text { implementar a lei recém revisada }\end{array}$ \\
\hline Norris A et al., $2011^{40}$ & $\begin{array}{l}\text { Abortion stigma: a } \\
\text { reconceptualization of } \\
\text { constituents, causes, and } \\
\text { consequences }\end{array}$ & Análise conceitual & $\begin{array}{l}\text { Conceituar e listar os elementos da } \\
\text { estigmatização }\end{array}$ \\
\hline Chełstowska A, $2011^{41}$ & $\begin{array}{l}\text { Stigmatization and } \\
\text { commercialization of } \\
\text { abortion services in Poland: } \\
\text { Turning sin into gold. }\end{array}$ & Analítico & $\begin{array}{l}\text { Discutir a vinculação entre restrições } \\
\text { legais e a privatização da atenção ao } \\
\text { aborto }\end{array}$ \\
\hline Lipp A, $2011^{42}$ & $\begin{array}{l}\text { Stigma in abortion care: } \\
\text { Application to a grounded } \\
\text { theory study }\end{array}$ & Analítico & $\begin{array}{l}\text { Discutir o estigma na prática da } \\
\text { enfermagem obstétrica como "dirty } \\
\text { work" }\end{array}$ \\
\hline
\end{tabular}

cial relacionada ao aborto; o segundo coloca em foco os indivíduos e/ou grupos afetados, analisando suas experiências, percepções e práticas; o terceiro discute lacunas entre as leis e as práticas assistenciais.

a) A discussão conceitual sobre estigma

$\mathrm{Na}$ literatura que se debruça na discussão conceitual sobre aborto e estigma ${ }^{7,8,10,11,30,32,34,40,42}$ é marcante a relevância dos estudos de Erving Goffman, principalmente de sua publicação Estigma - Notas sobre a manipulação da identidade deteriorada $a^{2}$. Desde essa perspectiva analítica, trabalha-se a proposição de que nos processos estigmatizantes os indivíduos são marcados por desgraça, vergonha e até mesmo repugnância, e o estigma estraga ou mancha suas identidades sociais, com o consequente efeito de excluí-los e desacreditá-los. As modalidades de estigma propostas por Goffman - as provenientes de deformidades corporais, as marcas de caráter e o estigma tribal - são revisitadas nos estudos analisados ${ }^{2}$. As duas primeiras situações podem estar associadas à mulher que engravida (as transformações corporais) e aborta (o caráter desnaturado da recusa da maternidade), e o estigma tribal institui a ideia de um coletivo de mulheres malditas: mães e esposas más e egoístas ${ }^{11}$. Outro artigo ${ }^{42}$ trabalha com as dimensões analíticas de "autoestigma" ou "estigma sentido", discutindo que o descrédito no caso de aborto provém de duas fontes: de um lado, da própria mulher que aborta que internaliza os preconceitos da sociedade; de outro lado, o estigma imputado pelo outro, cujas atitudes estigmatizam a mulher. $\mathrm{O}$ estigma opera um imaginário das mulheres que abortam como possuidoras de atributos e características que as tornam diferentes dos outros, desvalorizando-as ou depreciando-as aos olhos dos outros e da sociedade, ou seja, portadoras de uma "identidade deteriorada"10-12,25,26,31-34,40-44.

O estigma relacionado ao aborto é largamente reconhecido, mas pouco teorizado e pesquisa$\mathrm{do}^{40,44}$. As autoras argumentam que, embora seja um fato generalizado, a produção social do estigma é local e varia segundo as estruturas de desigualdades de poder; ressaltam a importância de considerar as normas sexuais e reprodutivas em seu contexto e compreender, em cada situação, como a transgressão dos estereótipos de gênero - o imperativo da maternidade, a pureza sexual feminina, o instinto de cuidar de outros - marca as mulheres como seres inferiores, deteriorados: promíscuas, pecadoras, sujas, assassinas. Sugerem que o estigma relacionado ao aborto é produzido, reproduzido e manifestado e deve ser analisado em diferentes níveis: no plano individual e das relações interpessoais e comunitárias, no plano das políticas governamentais e do sistema jurídico legal, no plano das macroestruturas econômicas e dos discursos culturais, entre outros. 
Trabalhos ${ }^{45}$ sobre o conceito de estigma aplicado a outros temas de saúde - como HIV/AIDS e doenças mentais - também são referencia para autores que discutem estigma e aborto $30,34,40,44$ Seguindo esses autores, Kumar et al. ${ }^{44}$ tematizam a natureza relacional do estigma e seu processo de produção: rotulação (as diferenças são identificadas e rotuladas); estereotipagem (crenças culturais dominantes associam os indivíduos rotulados a características negativas); separação (pessoas rotuladas são classificadas em categorias, que separam o "nós" e o "eles"); e discriminação (pessoas rotuladas experimentam perda de status, rejeição, exclusão e discriminação).

Alysson Lipp ${ }^{42}$ afirma que toda sociedade tem expectativas e projeções sobre seus membros, delineando o que Goffman ${ }^{2}$ chamou de identidade virtual. $\mathrm{O}$ aborto é estigmatizado porque viola projeções ideais do feminino e contraria normas sexuais e reprodutivas. Tecnologias médicas reforçam essas idealizações, como o ultrassom, por exemplo, ao atribuir personalidade ao feto e à mulher grávida o estatuto de "mãe"46.

Alguns textos ${ }^{11,26,30,34,44}$ detalham o componente psicológico e relacional do estigma ao aborto, em que o estigma perpassa a forma como indivíduos percebem a si e aos outros. As reações negativas identificadas/percebidas/presumidas nos outros pode influenciar as decisões quanto a revelar ou ocultar a situação estigmatizada, criar conflitos nos relacionamentos afetivos, impactar a saúde física e mental da pessoa e/ou atrasar ou evitar cuidados de saúde. Seguindo a conceitualização de Link et al. ${ }^{47}$, Schellenberg e Tsui ${ }^{26}$ analisam o estigma em três dimensões: estigma percebido, refere-se a percepção da mulher sobre ideias e sentimentos que terceiros têm sobre o aborto ou sobre a maneira que reagiriam sabendo que ela fez ou tem intenção de faze-lo; o estigma experimentado, refere-se a sofrer rejeição, discriminação ou outro prejuízo por causa das reações negativas dos outros - profissionais, parentes, amigos, vizinhos - ao saberem que a mulher realizou ou planeja realizar um aborto; o estigma internalizado, refere-se à incorporação de percepções, crenças e atitudes dos outros que são negativas para a sua autoavaliação, resultando em diminuição da autoestima ou sentimentos de culpa ou vergonha. O estigma refere-se à forma como indivíduos percebem os outros e o quanto as reações negativas identificadas (percebidas) nos outros pode atrasar ou evitar cuidados de saúde.

b) Indivíduos e grupos afetados

Três são os grupos afetados pelo estigma ${ }^{40}$ : (1) mulheres que abortam, (2) profissionais de saúde que prestam atenção ao aborto, e (3) os apoiadores das mulheres, como parceiros, familiares, amigos, defensores do aborto.

As informações sobre os efeitos do estigma relacionado ao aborto não são apreendidas em relatórios oficiais dos governos que, quase sempre, apresentam dados objetivos sobre mortalidade materna e outros temas de saúde da mulher, mas omitem as vozes e as experiências das mulheres. Porém, resultados apresentados nesta revisão apontam as experiências das mulheres e dos profissionais de saúde e refletem sobre o estigma do aborto, inclusive com estudos comparativos de diferentes países.

A dualidade calar/revelar sobressaiu na análise de vários estudos, que recuperam a discussão de Goffman ${ }^{2}$ sobre estigma e visibilidade: de um lado, estão os estigmas que são imediatamente visíveis para os outros e, de outro, aqueles que são invisíveis, a menos que sejam revelados, sendo estes últimos adjetivados como estigmas "conciliáveis"; eles não tornam o imediatamente desacreditado, mas “desacreditável”. Esse é o caso do aborto, cuja visibilidade depende da dinâmica segredo-revelação.

O estigma envergonha e faz calar quem decide interromper uma gravidez, bem como os prestadores da assistência ${ }^{11,48}$. A revelação do aborto ou da intenção de fazê-lo pode criar conflitos e a consciência disso pode influenciar a decisão da mulher sobre contá-lo ou não ao parceiro, à família, a amigos e ao profissional de saúde o acontecido ${ }^{25,26,49}$.

Major e Gramzow ${ }^{49}$ identificaram que, para as mulheres que abortam a necessidade de manterem segredo a este respeito era um motivo de sofrimento psicológico. Dez anos mais tarde, Kumar et al. ${ }^{44}$ descrevem este silencio como um verdadeiro ciclo vicioso: poucas mulheres revelam que fizeram um aborto; ideias padronizadas sobre o aborto como algo "raro" e "desviante" são reforçadas; incrementa-se a discriminação da prática de aborto; o medo da estigmatização aumenta e faz com que as mulheres se sintam pouco seguras para revelar que fizeram um aborto.

Também Harris ${ }^{22}$ enfatizou que o estigma pode levar uma mulher a manter sua decisão de abortar reservada para não macular seu status pessoal e preservar-se como uma pessoa "sem marca”. Shellemberg et al..$^{34}$ aponta o dilema das mulheres quanto à revelação de seus abortos atuais ou passados como um nó crítico em suas trajetórias de vida: uma em três mulheres que abortam antecipa um possível estigma, evita falar a respeito e vivencia a decisão de abortar como processos solitários em relação aos parceiros afetivos ou a familiares. 
Rossier ${ }^{50}$, no artigo "Aborto: um segredo aberto?" se pergunta como as mulheres mantêm em segredo o aborto? E o que propiciaria a elas revelá-lo? Com uma reflexão sociológica sobre o sigilo, a autora considera que este fornece um véu por trás do qual os atos proibidos, as violações legais, a ineficiência e a corrupção são ocultados. No caso dos abortos, o silencio não seria apenas o ocultamento de uma transgressão legal ou da realização de algo proibido, mas também uma estratégia para contornar conflitos de valores, julgamentos dos outros e processos de (auto) estigmatização e se manter dentro da ordem social estabelecida. Dois terços das mulheres da pesqui$\mathrm{sa}^{26}$ tinham a convicção de que seriam vistos pelas pessoas de seu convívio de um modo diferente se soubessem sobre seus abortos e mantinham-no sob segredo como um preventivo de estigmas. As mulheres com intuito de preservar sua imagem, ao abortar, buscam serviços clandestinos em locais cada vez mais distantes de sua moradia ${ }^{51}$.

Em culturas asiáticas ${ }^{23,28}$ e africanas ${ }^{37,52}$, o estigma do aborto relaciona-se a padrões culturais tradicionais que condenam a interrupção da gravidez fruto de relações sexuais pré ou extramarital. A perda fetal espontânea e a morte neonatal são ambos sinônimos de aborto, e a mulher é julgada como frágil ${ }^{52}$. Em diferentes contex$\operatorname{tos}^{20,23,28,29}$ não são as leis o fator de maior limitação, mas questões culturais religiosas e espirituais. No silêncio das que abortam, além das implicações emocionais de medo e culpa, sobressai o pecado como peso da condenação religiosa ${ }^{37}$.

Em estudos com grupos focais com mulheres adultas ${ }^{35,36}$, narrativas de jovens ${ }^{53-55}$ e outros populacionais com dados da Índia e $\mathrm{Cuba}^{56,57}$ foram ressaltadas a percepção sobre aborto e suas implicações socioculturais como determinantes do estigma.

Ainda no campo psicológico, o processo de internalizar ou de lidar com uma discriminação pode impactar diretamente a pessoa em sua autopercepção e levar à insegurança e à depressão $\mathrm{O}^{26,40,43}$. Com sentimentos de culpa, as mulheres vivenciam o aborto como uma "marca de caráter"11. Opostamente, em países onde os direitos individuais são mais prezados, como a Austrália, a vivencia do aborto pode ter expressão positiva: as mulheres: sentem-se com autonomia e competência suficiente para decidir sobre interromper ou não uma gravidez ${ }^{58}$.

Entre os autores que se dedicaram a teorizar os efeitos do estigma do aborto vivenciados pelos profissionais de saúde, destacam-se os trabalhos de Lisa Harris et al. que discutem o "paradoxo da legitimidade": profissionais que realizam o procedimento dentro de marcos legal, apesar de capacitados e legitimados, são vistos, inclusive pelos colegas, como desviantes, desqualificados, ilegítimos $^{19}$. Esses e outros pesquisadores ${ }^{19,30,59}$ atualizam as concepções de Hughes que denominou de "trabalho sujo", trabalhos estigmatizados como coveiro, lixeiro e outros; seus praticantes os "trabalhadores sujos" - executariam funções socialmente necessárias, mas consideradas tarefas materialmente repugnantes, socialmente degradantes ou moralmente duvidosas. Igualmente, os médicos envolvidos na atenção ao abortamento são considerados de menor qualificação técnica, "açougueiros", e o descrédito e a depreciação passam a ser a tônica quando se refere às práticas destes $^{18,19,40}$. A dinâmica do estigma do aborto como "trabalho sujo", pode estar relacionada, por um lado, ao contato físico com a genitália feminina e o sangue e partes fetais - como se os profissionais estivessem "maculados"; por outro lado, ao trato direto com uma pessoa estigmatizada, a mulher que aborta sobre a qual pesa uma mácula moral, possuidora de uma natureza desvirtuada, uma identidade deteriorada ${ }^{42}$. Para os profissionais, o desafio torna-se ainda maior quando estão envolvidos na atenção a abortos do segundo trimestre de gestação $0^{33}$.

Na literatura sobre estigma e aborto, há análises sobre a recusa do atendimento por objeção de consciência. Refere-se às crenças morais (culturais, religiosas) de cada um e associada a uma recusa de cuidados. Em certos cenários pode haver abusos na utilização da figura jurídica da objeção de consciência por medo de uma estigmatização pessoal ${ }^{9}$. Por igual motivo, os prestadores de serviços de aborto legal podem ser dissuadidos de anunciarem-se publicamente como tal, e adotarem eufemismos e serem conhecidos como "profissionais da saúde da mulher" . Entretanto, outros estudos refutaram as suposições de que profissionais evitariam prestar assistência por temerem reações de discriminação, violência ou assédio: há relatos de manifestações afirmativas por parte dos profissionais que prestam assistência à mulher que aborta, que consideram ser seu dever ético atender a essa população ${ }^{14,15,18}$. Os obstáculos à integração do aborto à prática médica seriam de outras ordens: o acesso limitado aos serviços por falta de profissionais treinados, desinformação das mulheres e barreiras administrativas (consentimento do marido, assinatura de vários médicos); a baixa qualidade da atenção por uso de tecnologias antiquadas como a curetagem e a falta de misoprostol, e atitudes hostis dos provedores; o ensino tecnicista com capacitação carecendo de espaços pedagógicos para revisão de 
mitos e valores sobre aborto; o quadro legal restritivo que influencia no número de abortos inseguros; e o estigma que, por associar sentimentos de culpa, vergonha e segredo, amplia as barreiras para a busca e a prestação dos serviços.

Um conjunto de publicações ${ }^{30,51,60}$ discorre sobre estratégias de alivio de tensões e pressões sofridas pelos profissionais que assistem as mulheres que abortam. Apontam que os indivíduos vulneráveis podem evitar, resistir, ou transformar o processo de estigmatização, ao empregarem técnicas de reenquadramento (alterar significados), calibragem (dar pesos distintos a certas praticas do trabalho) e de desfocagem (destacar os aspectos positivos do trabalho) e, assim, produzir espaços "seguros" para a manutenção de uma identidade profissional positiva. Aqueles que superaram os limites do silêncio imposto pelo receio de serem estigmatizados, sentem-se como que "saindo do armário", em alusão ao movimento de direitos dos gays ${ }^{30}$. A percepção dos "profissionais de consciência" de que estão comprometidos em fazer o melhor pelas mulheres, seria um exemplo de superação dos temores do estigma ${ }^{19}$.

Para apoiar as mulheres em suas experiências de aborto, Littman et al..$^{43}$ consideram a necessidade de se construir nos serviços uma 'cultura de suporte', com reflexão das questões socioculturais. Orientações para o uso autônomo dos comprimidos de misoprostol seria uma forma de empoderamento: a biotecnologia contribuindo para uma transição dos sentimentos das mulheres, do medo à segurança, ${ }^{8,33}$. Quando o enfoque psicológico está associado ao técnico na assistência, os profissionais de saúde podem contribuir para reverter os sentimentos de isolamento daquelas mulheres que abortam e que percebem atitudes sociais negativas dirigidas a elas ${ }^{21}$.

Uma revisão $0^{61}$ aborda práticas pedagógicas para lidar com os aspectos emocionais de diferentes temas de saúde e estigma, consideradas adaptáveis para apoiar mulheres estigmatizadas pelo aborto, enfatiza os efeitos positivos das técnicas de aconselhamento através de internet, do telefone e de espaços presenciais para troca de vivências.

c) Lacunas entre leis e práticas

Um grupo de artigos ${ }^{7-9,13,23,18,24,39,58,62-66}$ analisa arcabouços jurídicos, processos de revisão de leis e/ou obstáculos na implementação de políticas públicas de assistência ao abortamento previsto em lei. Analistas consideram que o estigma do aborto - "ato pecaminoso", "anti-natural", "prática assassina" - é um fator que limita e atrasa mudanças legais, uma vez que legisladores e outros atores políticos relutam em considerar os argu- mentos da saúde pública sobre as consequências do aborto inseguro e, muitas vezes, os debates giram em torno de argumentos morais e religio$\operatorname{sos}^{7,8,39,41,58,65,66}$.

A revisão de leis nacionais restritivas do aborto e a regulação da assistência por protocolos técnicos foram temas estudados no continente africano - Gana, Quênia, Nigéria, Senegal, África do Sul, Tanzânia e Zimbabwe. Desde 1996, sinalizaram esforços de juízes, médicos, gestores, parlamentares e da sociedade para reduzir sequelas e mortes do aborto inseguro e ampliar o acesso à atenção qualificada ${ }^{65,66}$. Narrativas de jovens que revelavam o conteúdo estigmatizador de suas condenações por aborto repercutiram nas estratégias para os que advogavam (advocacy) pela mudança da lei ${ }^{17}$. Nessa perspectiva, da análise de estudos empíricos sobre a representação das mulheres que abortaram, extraíram-se mensagens para entender e reverter o estigma do aborto ${ }^{16}$.

A aliança entre as associações profissionais e os movimentos feministas também construíram caminhos para superar os efeitos das proibições legais, enfrentar discriminações culturais, impulsionando mudanças no acesso e na qualidade da atenção, como no Programa Iniciativas Sanitárias do Uruguai ${ }^{18}$ e no Distrito Federal do México ${ }^{8}$.

Assim como as leis, a religião é relacionada no rol dos complexos determinantes que podem reforçar/desconstruir o estigma do aborto, e mereceu estudos de opinião entre católicos no México $^{27,38,67}$, além de análises de argumentos das campanhas de mídia realizadas na Índia ${ }^{24}$. Nos países em que a Igreja católica deposita excessivo valor na vida do feto, foi analisado que obstetras e enfermeiras vivenciam na assistência às mulheres que abortam, o dilema de refletir ou rejeitar os preceitos católicos. Para estes provedores, cuja formação profissional os qualifica para "a missão de salvar o feto"7,9, a realidade da demanda do abortamento exerce uma pressão sobre tal contradição, em que o simples ato de administrar o misoprostol nestes casos pode representar um ato transgressor e passível de temor de estigma pelo profissional que o aplica.

O estigma social foi demonstrado como um obstáculo ao acesso ao aborto seguro, mesmo onde o procedimento é previsto por lei com políticas e protocolos para o seu atendimento $7,8,13,18,39,41,58,62,65,66,68$. Esbarram nas ambiguidades e inseguranças dos profissionais na tradução prática da lei ${ }^{13}$, seja por sentirem como uma enorme responsabilidade o fato de terem que aplicar os critérios de elegibilidade e evitar possíveis consequências legais; seja por medo de serem rotulados pejorativamente como aborteiros e carre- 
garem o estigma dos agentes da morte ${ }^{7,13,19}$. Há ainda os que recusam assistência ao abortamento por temerem situações de violência de políticos e religiosos antiabortos, exemplificados pelos obstetras americanos ${ }^{60}$ e brasileiros ${ }^{25}$.

Vários autores discutem a responsabilidade ético-profissional de gestores e médicos nas omissões de atenção ao aborto legal e seguro $^{7-9,13,18,19,25,39}$. Referem-se, em particular, aos ginecologistas e obstetras, cujas atitudes estigmatizantes, somadas à discriminação social e de gênero, comprometem os cuidados com a saúde feminina. De Zordo ${ }^{25}$, entrevistando profissionais brasileiros, encontra que as mulheres mentiam que haviam sido estupradas para realizar o aborto previsto em lei, e outros evitavam informar sobre o direito ao aborto às vítimas de violência sexual. De outro lado, as mulheres, criam estratégias para cumprir as regras sociais falando dos abortos provocados de forma dissimulada como abortos espontâneos, fruto de acidentes, quedas ${ }^{69}$.

Artigo referente à sociedade estaduniden$\mathrm{se}^{44}$ concluiu que o fato da assistência ao abortamento ter ficado progressivamente à margem do conjunto da ginecologia-obstetrícia, tornando-se quase uma subespecialidade, é parte do processo de sua estigmatização. Igualmente, a fraca integração do tema nos currículos médicos e a não exposição dos estudantes a uma reflexão ético-profissional sobre direitos, corpo e reprodução dificultariam o florescimento de práticas de cuidado não estigmatizantes, livres de juízo de valor, mesmo em contextos onde o aborto é legalizado ${ }^{14,15,60}$. Analistas ${ }^{18}$ discutem a relação não linear entre a revisão das leis e o arrefecimento do estigma do aborto: uma maior liberalidade legal parece não necessariamente cessar o sentimento de culpa e de vergonha de muitas mulheres, fruto da desaprovação social e religiosa.

Finalmente, cabe notar como o debate sobre aborto e estigma sofreu uma transição ao longo do período analisado. O texto mais antigo abordou o estigma que circundava as mães com filhos ilegítimos na sociedade norteamericana, sendo o aborto um recurso das mulheres para escaparem às discriminações ${ }^{70}$. Em 1984, em estudo com universitários, pesquisadores verificaram que homens e mulheres manifestaram que manteriam distancia de mulheres que abortaram ${ }^{71}$. Em estudo de 1999, floresceram as implicações emocionais pela não revelação do aborto e as técnicas psicológicas de suporte às mulheres que abortavam $^{49}$. E em 2003, Ellison ${ }^{72}$ analisa a natureza autoritária das politicas reprodutivas norte-americana que modelavam as decisões das jovens solteiras segundo um padrão de castidade, sem espaço para a maternidade, empurrando-as para o aborto para fugirem do estigma da desonra que recai sobre elas. Mais tarde, um grupo de artigos enfocou o processo da revisão de leis do aborto em diferentes continentes (África, 2004; Polônia, 2008; México, 2010) e, mais recentemente, sobressaíram os estudos dedicados à teorização sobre estigma e aborto e às estratégias de proteção à vulnerabilidade de mulheres e profissionais envolvidos no abortamento (2008-2014).

Os resultados de trabalhos sobre estigma e HIV/AIDS ${ }^{73,74}$ têm sido fontes inspiradoras para o desenho de estudos sobre aborto dos anos 2000. Incorporaram as novas perspectivas teóricas sobre estigma, indo além de uma abordagem individual das marcas visíveis e direcionando a atenção às dinâmicas coletivas que atribuem às mulheres que abortam uma marca moral de "transgressoras" do papel de mãe ${ }^{44}$ - talvez, essa renovação conceitual da categoria estigma responda pela profusão de artigos publicados nos anos recentes.

\section{Considerações finais}

$\mathrm{Na}$ presente pesquisa, alguns artigos abordaram de forma mais analítica a interface aborto e estigma, enquanto outros utilizaram mais instrumentalmente a categoria estigma para analisar experiências de mulheres e/ou profissionais de saúde e conhecer o que tinham vivido como pensavam e percebiam e o que sentiam em suas experiências com o aborto.

Os autores procuraram mostrar que, justificado em parte pela natureza intima dos temas da saúde reprodutiva, o aborto tem se mantido em segredo ou em seletiva revelação, numa tentativa das mulheres que abortam de prevenir atitudes estigmatizantes e de serem marcadas por estereótipos $^{22,34,44,49,50}$. As abordagens qualitativas à temática do aborto abriram espaço para que se revelassem não só as experiências individuais de abortamento como os aspectos legais e da assistência. Identificaram que, para as mulheres que abortam, afloravam os sentimentos de culpa, baixa-estima, vergonha, medo e percebiam o cenário das unidades de saúde como um ambiente julgador e de indiferença às suas apreensões, demandas. Para o profissional, os temores eram as possíveis denuncias devido à ilicitude do procedimento de aborto.

Os analistas consideraram que a tensão que ocorre entre revelar $v s$. ocultar as situações de abortamento passa pela necessidade da mulher de manejar o julgamento de sua comunidade/ 
grupo social ou do serviço de saúde e as consequências da estigmatização. Tal interdição na comunicação sobre o aborto configura-se um obstáculo a mais e retém as mulheres na busca por atendimento. Hesitantes e temorosas da reação dos profissionais de saúde qualificados incrementam a cada dia as estatísticas dos abortos inseguros. Concorre para isto a reduzida divulgação dos serviços, o precário monitoramento da acessibilidade e qualidade do cuidado e a descontínua capacitação dos profissionais. E, nos países com leis restritivas, a revisão de fluxos da atenção e definição de normas e protocolos pode aprimorar a resposta à demanda por abortos seguros, redução da mortalidade materna e alcance das metas do Milênio (meta 5) ${ }^{62}$.

Com esse leque de questões reveladas pelos estudos, a categoria estigma tem sido um elemento/argumento decisivo para um maior entendimento e crítica dos aspectos sociais, médicos e legais da marginalização das mulheres adultas e adolescentes que abortam.

Nesse sentido, os profissionais de saúde jogam um papel fundamental de superação de certo "apartheid" da atenção ao abortamento nos serviços de saúde e nos espaços de formação profissional. Pesquisas locais demonstraram a recusa (velada ou explícita) de profissionais médicos ou de enfermagem de apoiar os procedimentos de interrupção da gravidez (administrar misoprostol ou preparar o instrumental para a aspiração manual intrauterina); do mesmo modo, observou-se que gestores flexibilizavam em demasia a utilização do dispositivo de objeção de consciência, trazendo risco à saúde física e mental das mulheres em abortamento ${ }^{7}$. Tais situações podem ter contribuído para empurrar a assistência ao aborto para fora dos hospitais/maternidades e serem operacionalizados em clínicas como uma subespecialidade, em países norte-americanos e europeus, ou em locais insalubres e clandestinos, como na América Latina.

A relação existente entre estigma social e leis restritivas do aborto foi objeto de estudo de alguns autores. Desde 1980, os artigos analisados trouxeram o contexto político e epidemiológico, assim como os avanços e limitações da atenção em Botswana, Burkina Faso, Canadá, Malásia, Mongólia, Paquistão, África do Sul, México, Uruguai, dentre outros. Ponderaram que para colocar em prática as mudanças ocorridas nos marcos legais, governos e ativistas feministas esbarravam com a posição de médicos e enfermeiros quanto à objeção de consciência ${ }^{7,9}$. Por outro lado, em iniciativas de mudanças no arcabouço jurídico do aborto, os médicos de "consciência" foram os que mais rapidamente aderiam ao novo status legal no México ${ }^{27,38,67,69}$ e África do Sul ${ }^{39,66}$, reforçando a análise de que em atendimentos controversos como o aborto, uma efetiva participação do profissional de saúde, é igualmente consciência-dependente. Dickens e Cook ${ }^{75}$ alertam que o compromisso de consciência é o reverso da objeção de consciência e pode superar os obstáculos culturais e religiosos para o cuidado da mulher em abortamento.

Pensando o estigma como uma construção histórica, as mudanças nas leis podem ser um impulsionador de transformações do ponto de vista cultural (e vice-versa), contribuindo para que o estigma também se modifique. A lógica proibicionista das sociedades, com leis retrógradas e abordagem religiosa conservadora, tem contribuído para o número de mortes maternas por abortamento e para a violação dos direitos de decidir das mulheres sobre sua reprodução. Nos trabalhos aqui analisados, o estigma tem permeado as atitudes, os discursos e as práticas das sociedades em relação a quem procura e a quem presta assistência à saúde sexual e reprodutiva: as práticas de contracepção e, em particular, a de emergência, os processos de esterilização voluntária, as fertilizações in vitro e os abortamentos ${ }^{7}$. No cotidiano dos serviços de saúde, gestuais e atos, ditos e não ditos, tem silenciosamente fermentado os processos de estigmatização. A compreensão de como operam essas "marcas invisíveis" que estão relacionadas ao estigma do aborto pode ser uma ferramenta importante para políticas de saúde e de direitos reprodutivos que se proponham a enfrentar o ciclo da gravidez indesejada-aborto clandestino, assim como assegurar de fato o acesso e a qualidade da assistência ao aborto em casos permitido por lei, e contribuir para o (re) desenho de ações para a redução de danos e a garantia dos direitos fundamentais das mulheres.

\section{Colaboradores}

L Adesse trabalhou na concepção e no delineamento do estudo, na pesquisa bibliográfica, análise dos dados, na redação e revisão crítica do artigo e na aprovação de sua versão final; CB Jannotti. trabalhou na concepção e no delineamento do estudo, na análise dos dados, na redação e revisão crítica do artigo e na aprovação de sua versão final. VM Fonseca e KS Silva trabalharam na redação e revisão crítica do artigo e na aprovação de sua versão final. 


\section{Referências}

1. Organização Mundial da Saúde (OMS). Abortamento Seguro: orientação técnica e de políticas para sistemas de saúde. 2a ed. Geneva: OMS; 2013.

2. Goffman E. Estigma - Notas sobre a manipulação da identidade deteriorada. Rio de Janeiro: Zahar; 1963.

3. Silveira OS, Martins LF, Soares RG, Gomide HP, Ronzani TM. Revisão sistemática da literatura sobre estigma social e alcoolismo. Estud. psicol. 2011; 16(2):131-138.

4. Parker R, Aggleton P. HIV/AIDS-related stigma and discrimination: a conceptual framework and implications for action. Soc Sci Med 2003; 57(1):13-24.

5. Adesse L. Assistência à mulher em abortamento: a necessária revisão de práticas de má conduta, preconceito e abuso. In: Deslandes S, organizador. Humanização dos cuidados em saúde: conceitos, dilemas e práticas. Rio de Janeiro: Fiocruz; 2006. p. 371-387.

6. Mendes KDS, Silveira RCCP, Galvão CM. Revisão integrativa: método de pesquisa para a incorporação de evidências na saúde e na enfermagem. Texto contextoenferm 2008; 17(4):758-764.

7. Cook R, Dickens BM. Reducing Stigma in Reproductive Health. Int J Gynaecol Obstet 2014; 125(1):89-92.

8. Lamas M. Between the stigma and the law: Legal abortion in Mexico. Salud Publica Mex 2014; 56(1):56-62.

9. Faúndes A, Duarte GA, Osis MJD. Conscientious objection or fear of social stigma. Int J Gynaecol Obstet 2013; 123(Supl. 3):S57-S59.

10. Kumar A. Everything is not stigma. Womens Health Issues 2013; 23(6):e329-e331.

11. Cockrill K, Nock A. I'm not that type of person: managing the stigma of having an abortion. Deviant Behav 2013; 34(12):973-990.

12. Cockrill K, Upadhyay UD, Turan J, Greene Foster D. Stigma of having an abortion: developing a scale and characteristics of women experiencing abortion stigma. Perspect. Sex Reprod Health 2013; 45(2):79-88.

13. Payne CM, Debbink MP, Steele EA, Buck CT, Martin LA, Hassinger JA, Harris LH. Why women are dying from unsafe abortion: narratives of Ghanaian abortion providers. Afr J Reprod Health 2013; 17(2):118-128.

14. Norman WV, Soon JA, Maughn N, Dressler J. Barriers to rural induced abortion services in Canada: findings of the British Columbia abortion providers. PLoS One 2013; 8(6):e67023

15. Dressier J. The perspective of rural physicians. PLoS One 2013; 8(6):e67070

16. Khan A. Induced abortion in Pakistan community-based research. J Pak Med Assoc 2013; 63(3):S27-32

17. Umuhoza C, Oosters B,van Reeuwijk M, Vanwesenbeeck I. Advocating for safe abortion in Rwanda: How young people and personal stories of young women in prison brought about change. Reprod Health Matters 2013; 21(41):49-56

18. Culwell KR, Hurwitz M. Addressing barriers to safe abortion. Int J Gynaecol Obstet 2013; 121(1):S16-S19.

19. Harris LH, Martin L, Debbink M, Hassinger J. Physicians, abortion provision and the Legitimacy paradox. Contraception 2013; 87(1):11-16.

20. Smith S. Reproductive health and the question of abortion in Botswana: a review. Afr J Reprod Health 2013; 17(4):26-34

21. Astbury-Ward E, Parry O, Carnwell R. Stigma abortion and disclosure. J Sex Med 2012; 9(12):3137-3147.
22. Harris LH. Stigma and abortions complications in the United States. Obstet Gynecol 2012; 120(6):1472-1474.

23. Tong W, Low WY, Wong YL, Choong SP, Jegasothy R. Exploring pregnancy termination experiences and needs among Malaysian women: A qualitative study. BMC Public Health 2012; 12(1):743

24. Banerjee SK, Andersen KL, Buchanan RM, Warvadekar J. Woman-centered research on Access to safe abortion services and implications for behavioral change communication interventions: A cross-sectional study of women in Bihar and Jharkhand, India. BMC Public Health 2012; 12(1):175.

25. De Zordo S. Representações e experiências sobre aborto legal e ilegal dos ginecologistas-obstetras trabalhando em duas maternidades em Salvador, Bahia. Cien Saude Colet 2012; 17(7):1745-1754.

26. Shellenberg KM, Tsui AO. Correlates of perceived and internalized stigma among abortion patients in the USA: an exploration by race and Hispanic ethnicity. Int J Gynaecol Obstet 2012; 118(2):152-159.

27. McMurtie SM, Garcia S. Public opinion about abortion -related stigma among Mexican Catholics and implications for unsafe abortion. Int J Gynaecol Obstet 2012; 118(2):S160-S166.

28. Levandowski BA, Kalilani-Phiri L, Kachale F, Awah PG, Mhango CK. Investigating social consequences of unwanted pregnancy and unsafe abortion in Malawi: the role of stigma. Int J Gynaecol Obstet 2012; 118(2):167-171.

29. Hosseini-Chavoshi M, Abbasi-Shavazi MJ, Glazebrook D, McDonald P. Psychological consequences of abortion in Iran. Int J Gynaecol Obste 2012; 118(2):S172-S177.

30. O’Donnell J, Weitz TA, Freedman LR. Resistance and vulnerability to stigmatization in abortion work. Soc Sci Med 2011; 73(9):1357-1364.

31. Gipson JD, Hirz AE, Avila JL. Perceptions and Practices of illegal abortion in Philippines Stud Fam. Plan 2011; 42(4):261-272

32. Harris LH, Debbink M, Martin L, Hassinger J. Dynamics of stigma in abortion work: Findings from a pilot study of the providers share workshop. Sol Sci Med 2011; 73(7):1062-1070.

33. Harris LH, Grossman D. Confronting challenge of unsafe second-trimester abortion. Int J Gynaecol Obstet 2011; 115(1):77-79.

34. Shellenberg KM, Moore AM, Bankole A, Juarez F, Omideyi AK, Palomino N, Sathar Z, Singh S, Tsui AO. Social stigma and disclosure about induced abortion: results from an exploratory study. Glob Public Health 2011; 6(1):111-125.

35. Tsui AO, Casterline J, Singh S, Bankole A, Moore AM, Omideyi AK, Palomino N, Sathar Z, Juarez F, Shellenberg KM. Managing unplanned pregnancies in 5 countries. Glob Public Health 2011; 6(Supl. 1):S1-S24.

36. Palomino N, Padilla MR, Talledo BD, Mazuelos CG, Carda J, Bayer AM. The social construction of unwanted pregnancy abortion in Lima, Peru. Glob Public Health 2011; 6(1):S73-S89.

37. Omideyi AK, Akinyemi AI, Aina OI, Adeyemi AB, Fadeyibi OA, Bamiwuye SO. Contraceptive practice, unwanted pregnancies and induced abortion in southwest, Nigeria. Int J Gynaecol Obstet 2011; 118(2):167-171. 
38. Rodríguez JV, Wilson KS, Olavarrieta CD, García SG, Sánchez Fuentes ML. Public opinion on abortion in eight Mexican States amid Opposition to legalization. Stud Fam. Plan 2011; 42(3):191-198.

39. Bateman C. Abortion practices undermining reformist laws - experts. S Afr Med J 2011; 101(5):302-304.

40. Norris A, Bessett D, Steinberg JR, Kavanaugh ML, De Zordo S, Becker D. Abortion stigma: a reconceptualization of constituents, causes, and consequences. Women's health issues 2001; 21(3):49-54.

41. Chelstowska A. Stigmatization and commercialization of abortion services in Poland: turning sin into gold. Reprod Health Matters 2011; 19(37):98-106.

42. Lipp A. Stigma in abortion care: Application to a grounded theory study. Contemp Nurs 2011; 37(2):115-123.

43. Littman LL, Zarcadoolas C, Jacobs AR. Introducing abortion patients to a culture of support: A pilot study. Archives of Women's Mental Health 2009; 12(6):419431.

44. Kumar A, Hessini L, Mitchell EMH. Conceptualizing abortion stigma. Cult Health and Sex 2009; 11(6):625639.

45. Bruce L, Jo CP. Conceptualizing stigma. Annual Review Soc 2001: 27(1):363-385.

46. Kirklin D. The role of medical imaging in the abortion debate. J Med Ethic 2004; 30(5):426.

47. Link BG, Struening EL, Rahav M, Phelan JC, Nuttbrock L. On stigma and its consequences: evidence from a longitudinal study of men with dual diagnoses of mental illness and substance abuse. J. Health Soc. Behav 1997; 38(2):177-190.

48. Harris LH. $2^{\text {nd }}$ trimester: breaking the silence and changing the disclosure. Reprod Health Matters 2008; 16(31):74-81.

49. Major B, Gramzow RH. Abortion as stigma: cognitive and emotional implications of concealment. J Pers Soc Psychol 1999; 77(4):735-745.

50. Rossier C. Abortion: An Open Secret? Abortion and Social Network Involvement in Burkina Faso. Reprod Health Matters 2007; 15(30):230-238.

51. Weitz TA, Cockrill K. Abortion Clinic Patient's opinion obtaining abortion. Patient Educ Couns 2010; 81(3):409-414.

52. Haws RA, Mashasi I, Mrisho M, Schellenberg JA, Darmstadt GL, Winch PJ. These are not good things for other people to know: How rural Tanzanian women's experiences of pregnancy loss and early neonatal death may impact survey data quality. Soc Sci Med 2010; 71(10):1764-1772.

53. Dahlbäck E, Maimbolwa M, Kasonka L, Bergström S, Ransjö-Arvidson AB. Unsafe induced abortion among adolescents in Lusaka. Health Care Women Int 2007; 28(7):654-676.

54. Atuyambe L, Mirembe F, Johansson A, Kirumira EK, Faxelid E. Experiences of pregnant adolescents voices from Wakiso, Uganda. Afr. Health Sci 2005; 5(4):304309.

55. Varga CA. Pregnancy termination among South African adolescents. Stud Fam Plan 2002; 33(4):283-298.

56. Ganatra B, Hirve S. Induced abortions among adolescent women in rural Maharashtra, India. Reprod Health Matters 2002; 10(19):76-85.

57. Peláez MJ, Rodriguez IA, Lammers C, Blum RWJ. Abortion among adolescents in Cuba. J Adolesc Health 1999; 24(1):59-62.
58. Wainer J. Abortion and the struggle to be good in the 1970s. Aust N Z J Psychiatry 2008; 42(1):30-37.

59. Chiappetta-Swanson C. Dignity and dirty work: nurses experiences in managing genetic termination for fetal anomaly. Qual Sociol 2005; 28(1):93-116

60. Freedman L, Landy U, Darney P, Steinauer J. Obstacles to the integration of abortion into obstetrics and gynecology practice. Perspect Sex Reprod Health 2010; 42(3):146-151.

61. Upadhyay UD, Cockrill K, Freedman LR. Informing abortion counseling: an examination of evidence-based practices used in emotional care for other stigmatized and sensitive health issues. Patient Educ Couns 2010; 81(3):415-421.

62. Singh. S. Global consequences of unsafe abortion. Women's Health 2010; 6(6):849-860.

63. Hessini L. Islam and abortion: the diversity of discourses and practices. IDS Bulletin 2008; 39(3):18-27

64. Billings DL. Scaling-up a public health innovation: a comparative study of post-abortion care in Bolivia and Mexico. Soc Sci Med 2007; 64(11):2210-2222.

65. Hord C, Wolf M. Breaking the cycle of unsafe abortion in Africa. Afr J Reprod Health 2004; 8(1):29-36.

66. Brookman-Amissah E, Moyo JB. Abortion Law reform in sub-saharan, Africa. Reprod Health Matters 2004; 12(24):227-234

67. Garcia SG. Policy implications of a National Public opinion survey on abortion in Mexico. Reprod Health Matters 2004; 12(24):65-74.

68. Dalvie SS. Second trimester abortion in India. Reprod Health Matters 2008; 16(31):37-45.

69. Erviti J, Castro R, Collado A. Strategies used by low-income Mexican women to deal with miscarriage and spontaneous abortion. Qual Health Res 2004; Oct; 14(8):1058-1076

70. Cutright P. Historical and contemporary trends in illegitimacy. Arch Sex Behav 1972; 2(2):97-118.

71. Weidner G, Griffitt, W. Abortion as a stigma: In the eyes of the beholder. J Res Pers 1984; 18(3):359-371

72. Ellison MA. Authoritative knowledge and single women's unintentional pregnancies, abortions, adoption, and simple motherhood: social stigma and structural violence. Med Anthropol Q 2003; 17(3):322-347.

73. Garcia S, Koyama MAH. Stigma, discrimination and HIV/AIDS in Brazil context 1998-2005. Rev Saude Publica 2008; 42(1):72-83.

74. Monteiro S, Villela WV, Knauth D. Discrimination, stigma, and AIDS: a review of academic literature produced in Brazil (2005-2010). Cad Saude Publica 2012; 28(1):170-176.

75. Dickens BM, Cook RJ Conscientious commitment to women's health. Int J Gynaecol Obstet 2011; 113(2):163166

Artigo apresentado em 30/04/2015

Aprovado em 13/08/2015

Versão final apresentada em 15/08/2015 